\title{
Insights into the Connectivity of the Human Brain Using DTI
}

\author{
S Kollias
}

\author{
Institute of Neuroradiology, University Hospital of Zurich, Switzerland.
}

\begin{abstract}
Diffusion tensor imaging (DTI) is a neuroimaging MR technique, which allows in vivo and non-destructive visualization of myeloarchitectonics in the neural tissue and provides quantitative estimates of WM integrity by measuring molecular diffusion. It is based on the phenomenon of diffusion anisotropy in the nerve tissue, in that water molecules diffuse faster along the neural fibre direction and slower in the fibre-transverse direction. On the basis of their topographic location, trajectory, and areas that interconnect the various fibre systems of the mammalian brain are divided into commissural, projectional and association fibre systems. DTI has opened an entirely new window on the white matter anatomy with both clinical and scientific applications. Its utility is found in both the localization and the quantitative assessment of specific neuronal pathways. The potential of this technique to address connectivity in the human brain is not without a few methodological limitations. A wide spectrum of diffusion imaging paradigms and computational tractography algorithms has been explored in recent years, which established DTI as promising new avenue, for the non-invasive in vivo mapping of structural connectivity at the macroscale level. Further improvements in the spatial resolution of DTI may allow this technique to be applied in the near future for mapping connectivity also at the mesoscale level.
\end{abstract}

Key words: Diffusion Tensor Imaging, human brain, white matter anatomy

\section{Definitions}

Diffusion tensor imaging (DTI) is a neuroimaging MR technique, which allows in vivo and non-destructive visualization of myeloarchitectonics in the neural tissue and provides quantitative estimates of $\mathrm{WM}$ integrity by measuring molecular diffusion. It is based in the phenomenon of diffusion anisotropy in the nerve tissue, in that water molecules diffuse faster along the neural fiber direction and slower in the fibertransverse direction (Fig. 1) The hindrance of water diffusion in white matter is putative due to the diffusion barrier represented by the cell membrane and the myelin sheath however, it is governed by several other, yet not completely elucidated, parameters related to the diffusion physics and biophysical properties of the tissue such as, the cell membrane permeability and the free diffusion coefficients for the cellular compartments.

The anisotropic diffusion of coherently oriented axonal fibers can be exploited for anatomic mapping and quantitative characterization of white matter tracts. This is done by depicting and measuring the diffusion tensor within the imaging voxels of the diffusion acquisition. Provided that 6 diffusion-encoded images sets are acquired along noncollinear directions, the diffusion tensor can be calculated. Diffusion tensor, is

Correspondence to: Prof. Dr. med. Spyros Kollias, Chief of MRI, Institute of Neuroradiology, University Hospital of Zurich, Switzerland

E-mail: kollias@dmr.usz.ch 


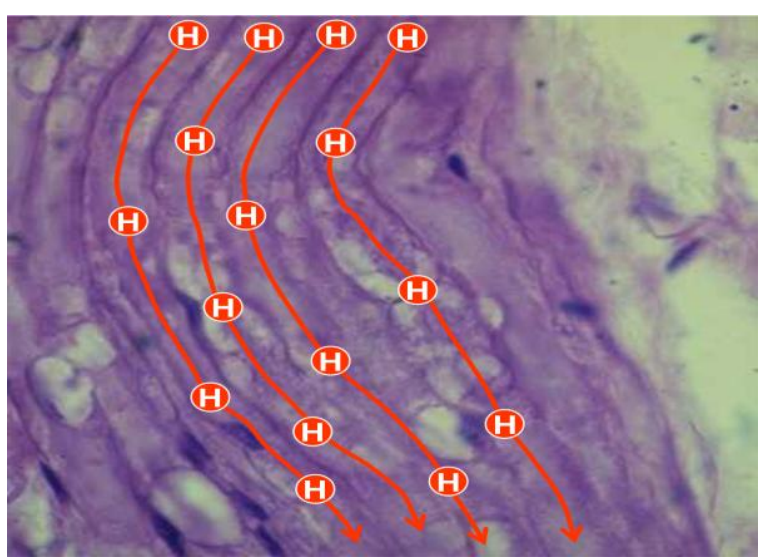

Figure 1: Schematic representation of water proton anisotropic diffusion along the long axis of myelinated white mater fibers. The displacement of the protons in the $3 \mathrm{D}$ space is faster along the direction of the fibers. Estimation of the direction of faster diffusion indicates the

a mathematical model of the 3D pattern of diffusion trsjectories (a 3X3 matrix of vectors) within the white matter tracts. The three principal axes of the diffusion tensor are termed the eigenvectors. The largest eigenvector is termed the primary eigenvector and indicates the direction and magnitude of greatest water diffusion. It is important for fiber tractography because it indicates the orientation of axonal fiber bundles. The degree of directionality of intravoxel diffusivity is measured by the fractional anisotropy (FA) index. When the value of the primary eigenvector is higher than the values of the second and third eigenvectors the FA will be high, indicating diffusion along a preferred direction. The graphic representation of the three eigenvectors corresponds to a prolate shape of diffusion ellipsoid with the long axis of the ellipsoid indicating the preferred direction of diffusion (Fig. 2). Its value varies from 0 (isotropic diffusion) up to maximum 1, indicating perfectly linear diffusion along the primary eigenvector.

\section{Visualization of white matter architecture using DTI}

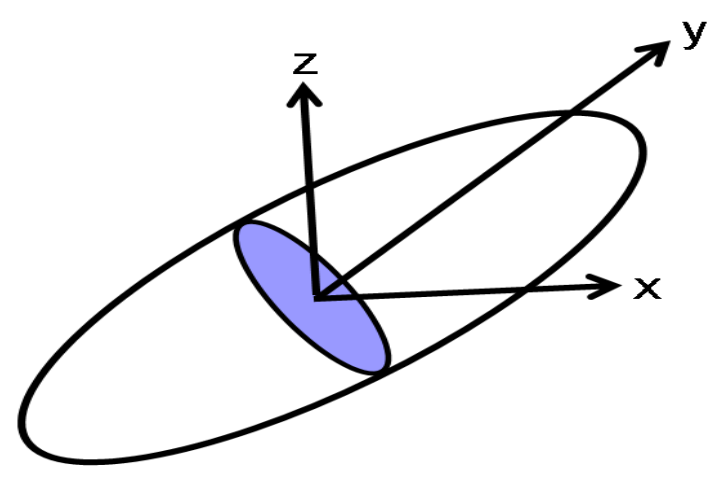

Figure 2: graphic representation of the diffusion ellipsoid. The long axis of the ellipsoid indicates the preferred direction of diffusion. Its value varies from 0 (isotropic diffusion) up to maximum 1 , indicating perfectly linear (anisotropic) diffusion along the primary eigenvector.

The fiber orientation information inherent in the primary eigenvector can be visualized on 2D images by assigning a color to each of the 3 mutually orthogonal axes, typically red for left-right, green for anteroposterior and blue for superior-inferior. The orientation of the primary eigenvector within each voxel gives the color in the specific voxel (Fig. 3). This way, white matter tissue can be further parcellated into specific white matter tracts according to their orientation in the 3D space (Fig. 4).

The orientation information can also be used for 3D fiber tracking of specific white matter tracts (Fig 5). The objective of fiber tracking is to determine intervoxel connectivity by using the diffusion tensor of each voxel to follow an axonal tract in 3D from voxel to voxel through the neural tissue. Several fiber tracking algorithms have been developed over the last years for anatomical reconstruction and quantitative characterization of white matter tracts. Grossly, these algorithms can be divided into deterministic and probabilistic methods. Deterministic, "streamline" tractography, infers the continuity of fiber bunbles from voxel-to-voxel. User-defined regions of interest, as well as constrains on the maximum turning angle of the fiber 
trajectory between voxels and on the minimum FA within a voxel for propagation of the streamline, are applied to restrict the fiber tracts to a specific pathway based on prior anatomical knowledge and thus to create realistic representations of the white matter pathways. Probabilistic methods, incorporate the expected uncertainty into the tracking algorithm and can be used to produce a connectivity metric for each voxel. They focus on the connectivity probabilities rather the actual WM pathways between voxels. Definition of a "probability density function" allows estimation of the uncertaintity of both intravoxel orientation and its propagation. These algorithms have the potential to delineate a greater portion of a white matter tract.
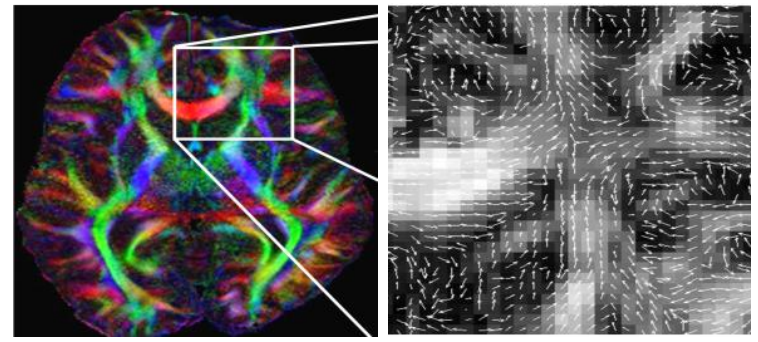

Figure 3: 2D DTI color-coded according the orientation in the $3 D$ space of the primary eigenvector within each voxel of the image (red for left-right, green for anterior-posterior and blue for superior-inferior). The direction of the main eigenvector indicates the fiber orientation within the specific voxel.

\section{Methods to improve determination of the diffusion tensor}

Much progress has also been achieved over the last years in improving the acquisition schemes of DTI data collection for better characterization of the water molecule diffusion. These include: a) increasing the angular resolution of the diffusionsensitizing gradients, b) performing a direct measurement of water diffusion in all directions and at different gradient strengths, referred to as Diffusion Spectrum Imaging

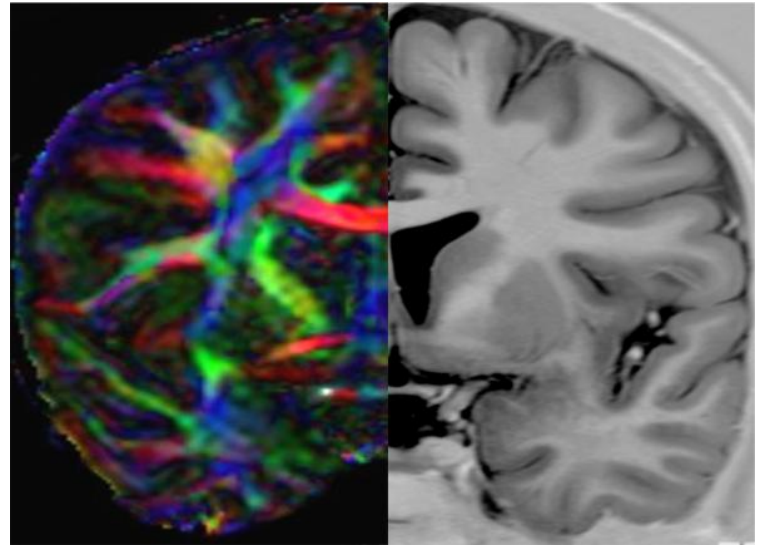

Figure 4: 2D DTI overlaid on a coronal inversion recovery (IR) anatomical MR image. Large white matter tracts of the projectional (blue), commissural (red) and association (green) fiber systems are distinguished from each other on the DT image.

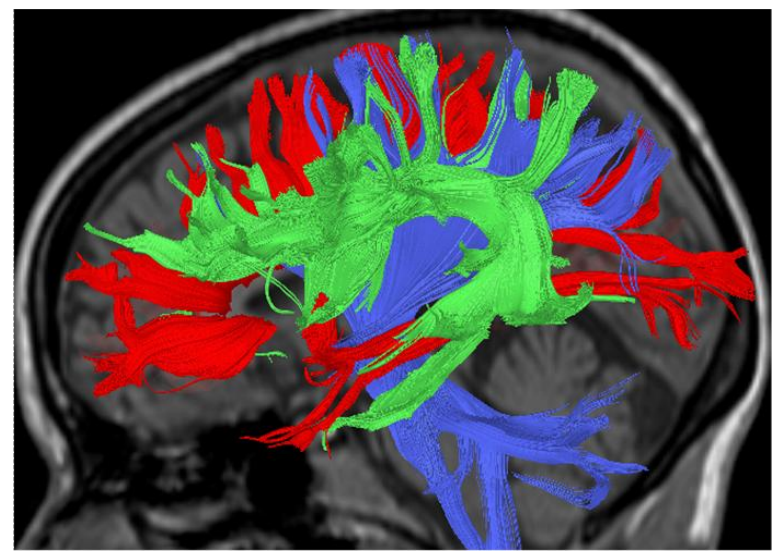

Figure 5: 3D reconstruction of fiber systems using the Fiber Assignment by Continuous Tracking (FACT) algorithm. Based on the localization and the specific main orientation we can virtually reconstruct individual fiber systems of the WM in great detail. We indentify the mediolaterally oriented commisural corpus callosum (red) the vertically oriented projectional systems of the corona radiata and internal caspule (blue) and the anteroposterior oriented long association fiber systems of the superior occipito-frontal fasciculus and arcuate fasciculus (green).

(DSI) although, this approach is time consuming and difficult to transfer in the clinical setting, c) performing measurements at many directions but one gradient strength only, referred as Q-ball imaging, with an acquisition time more compatible with clinical requirements. Certainly, the quality 
of the DTI is greatly facilitated by the use of higher field strength magnets (3-7 T), the use of specialized surface coils and the development of parallel imaging acquisition schemes.

The clinical and scientific utility of DTI fiber tracking is found not only in the anatomical delineation and localization of specific neuronal pathways but also in their quantitative assessment. Quantitative analysis examines the microstructure of the tracts according to the underlying FA values in the voxels contained within the tracts.

Topographic-anatomic classification of major fiber systems in the cerebral white matter of the primate brain

On the basis of their topographic location, trajectory, and areas that interconnect the various fiber systems of the mammalian brain are divided into: a) commissural, b) projectional and c) association fiber systems. On the basis of the known functional role of the areas that are interconnected by a fiber pathway, we can also make some general annotations regarding the possible functional role of the specific fiber system:

a) Commissural fiber systems: are bihemispheric, interconnecting cortical areas between the two hemispheres. The two major commissural fiber systems in the mamamalian brain are the corpus callosum and the anterior commissure. The fornix also has a small commissural component (hippocampal commissure) however, due to its predominant association fibers is included in the association fiber systems. The commissural system plays an important role in interhemispheric functional integration communicating perceptual, cognitive, learned, and volitional information. The corpus callosum facilitates interhemispheric interactions and integration. It is important for the performance of visual and tactile tasks that require transfer of sensory information between the cerebral hemispheres. It was demonstrated that anterior and body callosal connections are essential to perform temporally independent bimanual finger movements, whereas the posterior corpus callosum plays an important role in visual and visuospatial integration. Moreover, CC plays an important role higher order cognition including social, attentional and emotional well functioning and has been the focus of intense research in certain psychiatric disorders such as schizophrenia, autism, Tourette's syndrome, attention deficit hyperactivity disorder (ADHD), dyslexia, depression, genetic disorders (i.e., Down's syndrome), and demyelination (MS, HIV).

Corpus Callosum (CC): is the largest connective structure in the brain. It consists of over 200-300 million axons that transfer information between the two cerebral hemispheres. The interhemispheric connections between major neopalial portions of the two hemispheres are in the majority homotopic however, heterotopic connections also exist. There has been a debate whether the connections are primarily excitatory (integrating information across hemispheres) or inhibitory (allowing the hemispheres to inhibit each other in order to maximize independent function) but the consensus seems to be that the connections are primarily excitatory. The fiber distribution in the CC shows an anteroposterior topographical organization that is consistent with the topography of the cerebral cortex and is particularly precise in primates. Connections of the prefrontal cortex are located within the genu and anterior part of the body, premotor cortical connections in the midbody region, immediately posterior are the M1 and S1 tracts, and immediately posterior to $\mathrm{S} 1$ tracts are posterior parietal cortical connections. Tracts connecting the temporal and occipital cortices occupy the splenium of the CC (Fig $6 \mathrm{~A}$ and B). A more detailed topographical - 
somatotopical organization has been recently reported by Wahl et al., showing cortical motor fibers connecting defined body representations of $\mathrm{M} 1$ crossing the $\mathrm{CC}$ onto somatotopically organized circumscribed regions. Another important finding of this study was that the FA of the hand fibers correlated linearly with interhemispheric inhibition, induced by TMS, which suggested that microstructural information as measured by DTI can be directly linked to functional connectivity.
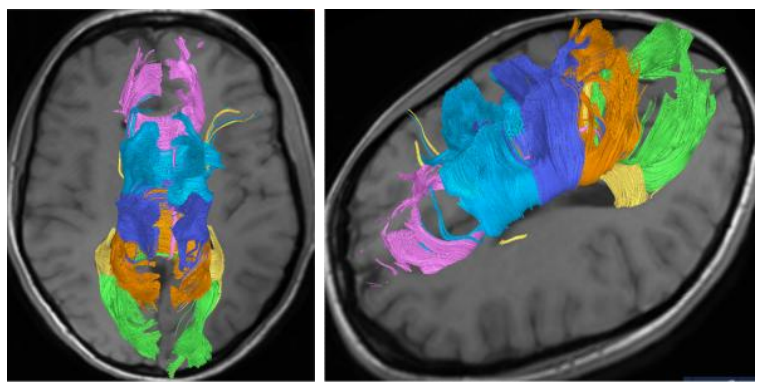

Figure 6: Lateral (A) and superior (B) view of DTI reconstructions of the corpus callosum. Connections of the prefrontal cortex (purple) are located within the genu and anterior part of the body, premotor cortical connections (cyan) in the midbody region, immediately posterior are the M1 and S1 tracts (blue), and immediately posterior to S1 tracts are posterior parietal cortical connections (magenta). Tracts connecting the temporal (yellow)and occipital (green) cortices occupy the splenium of the $\mathrm{CC}$.

Anterior commissure $(A C)$ : is a familiar landmark on sagittal and coronal conventional MR images where it crosses the midline as a compact cylindrical bundle between anterior and posterior columns of the fornix beneath the septum pelucidum and anterior to the third ventricle, shaped like the handlebars of a bicycle. On DTI reconstructions two types of fibers can be recognised: a) olfactory fibers, connecting the olfactory bulb, anterior olfactory nucleus and anterior perforated substance, and b) non-olfactory fibers, which are further subdivided in anterior subdivisions connecting the amygdale and temporal pole (following the course of the uncinate fasciculus), and more posterior subdivisions connecting the inferior temporal, parahippocampal and fusiform gyri as well as the inferior occipital cortex (following the course of the inferior longitudinal fasciculus) (Fig. 7).

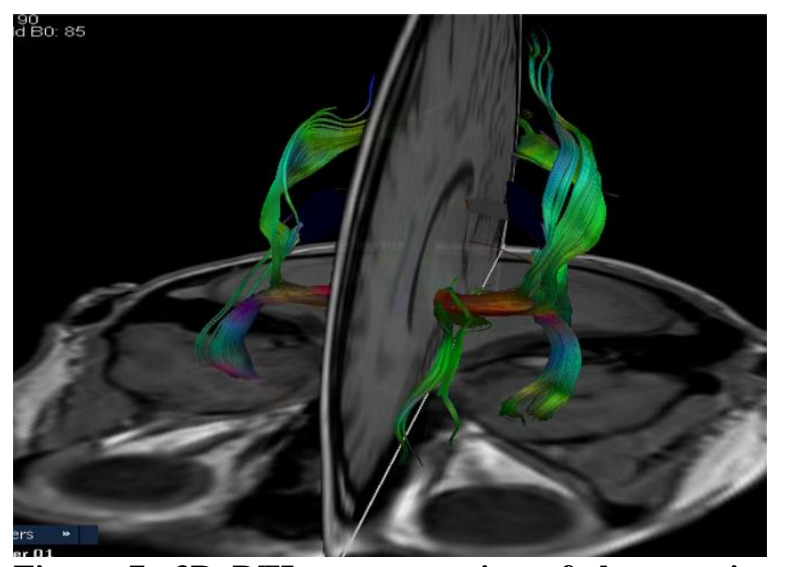

Figure 7: 3D DTI reconstruction of the anterior commissure including its anterior, olfactory, as well as its anterior and posterior, non-olfactory fiber subcomponents.

b) Projectional fiber systems: include the internal capsule, the coronal radiation including the corticospinal tract, and the geniculocalcarine tract.

Internal capsule (IC): The majority of connections between the cerebral cortex and subcortical structures travel through the internal capsule. It is composed of two major components: a) thalamic radiations, which radiate anteriorly to frontal cortex, superiorly to parietal cortex, posteriorly to parietal and occipital cortex, and inferolateraly to temporal cortex and b) motor projections connecting frontoparietal areas with subcortical nuclei and the spinal cord. The topographic organization of tracts within the IC is related to the anteroposterior part of their cortical connections irrespective from their afferent or efferent nature. The anterior limb carries thalamic projections to the frontal lobes and fronto-pontine motor fibers, the genu contains thalamic projections to the parietal lobe and corticonuclear motor projections, and the posterior limb contains 
posterior and inferolateral thalamic projections and corticospinal, corticopontine, and corticotegmental motor fibers (Fig 8).

Coronal Radiation and Corticospinal Tracts (CS): the coronal radiation is not a specific

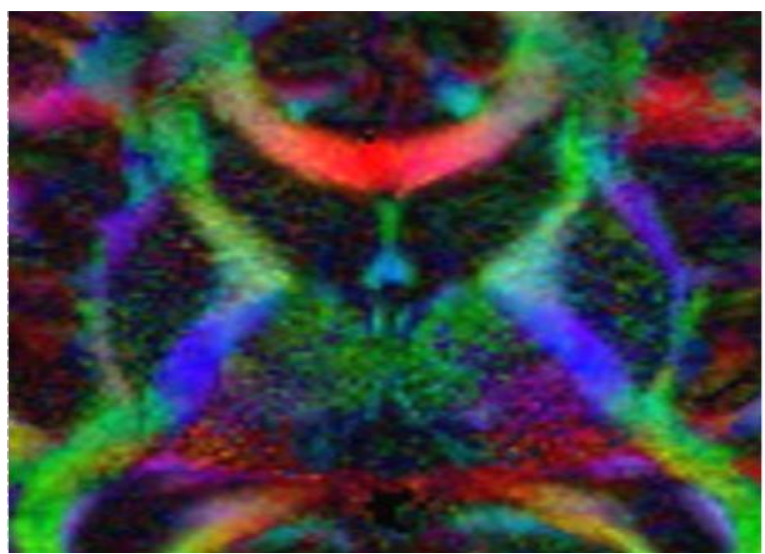

Figure 8: 2D DTI of the internal capsule. The different colours in the anterior limb, genu and posterior limb indicate the specific orientation of the thalamic radiations and motor projections connecting various areas of the cerebral cortex with subcortical structures. The most prominent structure is the corticospinal tract in the posterior limb coloured in dark blue, which indicates its more vertically oriented fibers.

tract per se, however, is one of the most easily identified structures on directional DTI color maps due to its coronally directed fibers that give him a distinct color (blue) from the adjacent tracts, which are primarily directed left-right (i.e., CC) or anteriorposterior (i.e., SLF) (Fig. 4). The corticospinal tract is easily reconstructed within the coronal radiation connecting primary motor areas with the spinal cord and passing through the internal capsule (Fig 9). In comparison with non-human primates, the sensorimotor tracts in humans are shifted more posterior (in the posterior third of the posterior limb) as a consequence of the prominent development of the prefrontal cortex and its projections through the IC. There is a small asymmetry between left and right CS tracts however, it tends to be small in healthy individuals and is not significantly different from zero across individuals.

Geniculocalcarine tract: the human visual system comprises elongated fiber pathways that represent a serious challenge for DTI. Reconstruction of the optic nerves and chiasm are compromised by the adjacent sinuses, eye muscles and bone. Crossing of the fibers in the optic chiasm represents also a challenge for traditional deterministic

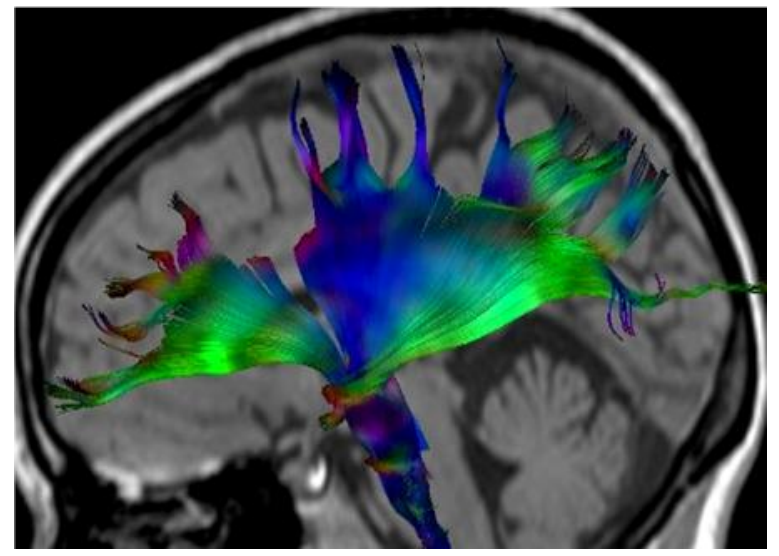

Figure 9: 3D DTI reconstruction of the coronal radiation. The blue-coloured fibers indicate the more vertically oriented fibers of the corticospinal tract.

reconstruction techniques. The optic radiation connects the LGN to the occipital (primary visual) cortex. The optic radiation is dissected into: a) the anterior ventral bundle (Meyer's loop), running anterior around the tip of the temporal horn and then passing posterior along the lateral wall of the ventricle to terminate on the lower lip of the calcarine fissure, b) the central bundle, leaving the LGN in a lateral direction and then turning posterior along the lateral wall of the ventricle to radiate into the visual cortex, and c) the dorsal bundle, running straight into a posterior direction forming a thick compact lamina terminating posteriorly in the upper calcarine lip. (fig. 10) 


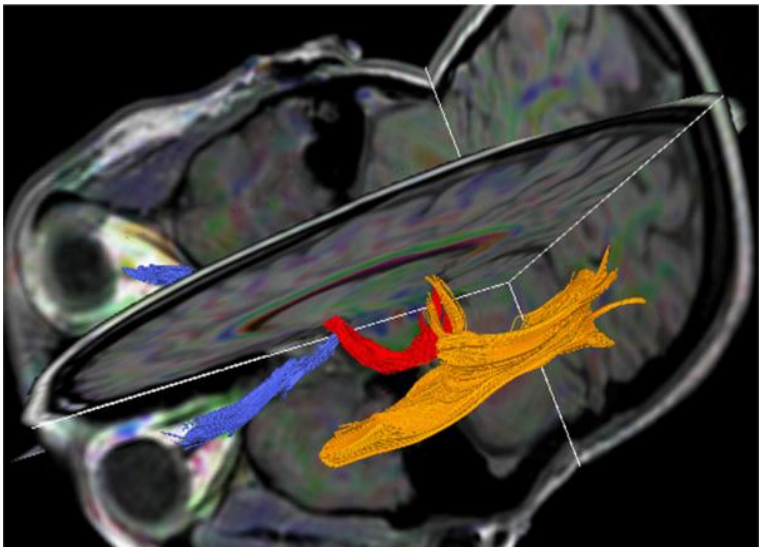

Figure 10: 3D, DTI reconstruction of the human visual system including optic nerves (blue), tracts (red) and optic radiations (magenta). The anterior ventral bundle (Meyer's loop), the central bundle, and the dorsal bundle, have different trajectories connecting the LGN with the occipital cortex.

c) Association fiber systems: interconnect cortical areas in each hemisphere and define large scale networks that subserve cognitive functions. They can be grossly divided into short and long association fiber systems. Short association fibers connect cortical areas around of a sulcus within the same lobe or in adjacent lobes (Fig. 11). They are most notable in the frontal and lateral occipital lobes in proximity to the long association superior and inferior longitudinal fascicles, which suggests that they could be part of that fascicles, which are known to consist of both short and long-range axonal fibers. The long association fibers form compact white matter bundles establishing distant interlobar connections. Understanding the long association pathways that convey cortical connections is a critical step in exploring the anatomic substrates of cognition in health and disease. The following long association fiber systems can be routinely reconstructed using DTI methodology.

Superior longitudinal fascicle (SLF) and arcuate fascicle $(A F)$ : the SLF and AF have historically been regarded by Burdach and Dejerine as a single fiber bundle in the human brain probably due to the inability of gross neuropathology to differentiate the two tracts. They used both names

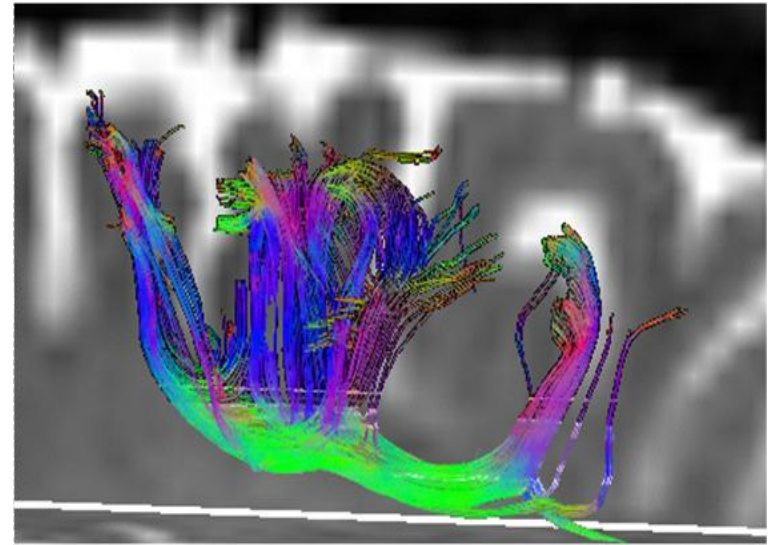

Figure 11: selective reconstruction of short association fibers around the central sulcus.

interchangeably in their descriptions. Nonhuman primate studies showed that these are two separate entities. Traditionally the SLF is considered to be a major association fiber pathway that connects the postrolandic regions (parietotemporal association areas) with the frontal lobe and vice versa. It stems from the caudal part of the superior temporal gyrus, arches around the sylvial fissure and advances forward to end within the frontal lobe. Recent, detailed DTI studies in monkeys and humans identified three subcomponents of the SLF: subcomponent I) located more suberior and medial, it courses in the white matter of the superior parietal and superior frontal lobes, subcomponent II) courses from the caudal part of the inferior parietal lobule to the distal premotor and prefrontal cortex, and subcomponent III) is identified in the opercular white matter of the parietal and frontal lobes extending from the inferior parietal lobule to the ventral premotor and prefrontal cortex (Fig 12).

The SLF by connecting the superior parietal lobule (important for limb and trunk location in body-centered coordinate space) with premotor areas (engaged in higher aspects of motor behaviour) is relevant to higher order control of body-center action and the initiation of motor activity. By connecting the inferior parietal lobule (concerned with visual spatial information) with the posterior prefrontal cortex (important for perception 
and awareness) is relevant in spatial attention. By connecting the supramarginal gyrus (concerned with higher order somatosensory information) with the ventral premotor area (containing mirror neurons for action imitation) is relevant to gestural components of language and orofacial working memory.

The AF originates in the cortex of the posterior ventrolatelar frontal lobe, passing backward to the inferior parietal lobe, where it arches around the lateral fissure to terminate in the posterior part of the superior and middle temporal gyrus (Fig 13). The ventral portion of the frontoparietal segment runs dorsal to the external capsule and the insular cortex. Wernike postulated the existence of a direct connection between the fronto-opercular, speech production, area (Broca's) and the posterior temporal, auditory word processing, area (Wernike's) and that a lesion of this theoretical pathway would cause an aphasia characterized by normal language comprehension and fluent speech but inability to repeat what had just been heard. The AF fasciculus was first described by Burdach and later confirmed by Dejerine who referred to the pathway as Burdach's arcuate fasciculus. Through this pathway the lateral prefrontal cortex receives auditory spatial information. Therefore the AF provides the means by which the prefrontal cortex can receive and modulate audiospatial information. Using DTI, Catani et al. showed that beyond the classical arcuate pathway connecting Broca's and Wernicke's areas directly, there is also an indirect pathway passing through the inferior parietal cortex. The indirect pathway runs parallel and lateral to the classical arcuate fasciculus and is composed of an anterior segment connecting Broca's territory with the inferior parietal lobe and a posterior segment connecting the inferior parietal lobe to Wernicke's territory. This model of two parallel pathways may explain the diverse clinical phenotypes of conduction aphasia.

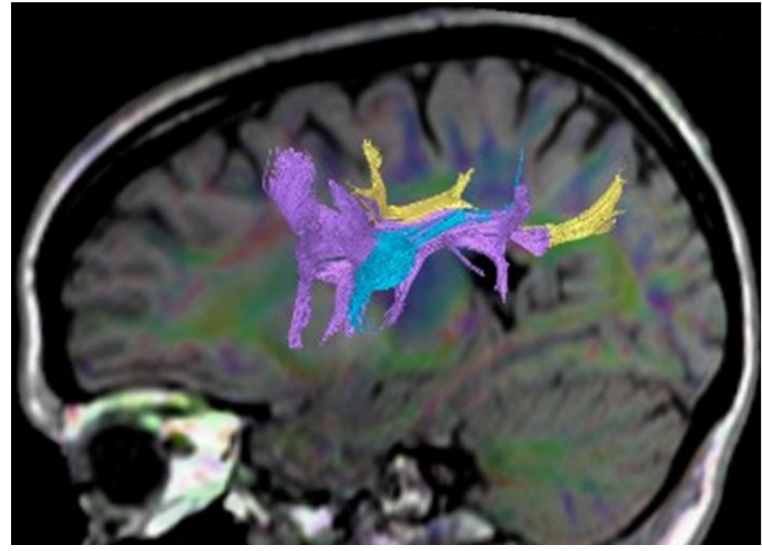

Figure 12: DTI reconstruction of the SLF. The three subcomponents (I: yellow, II: violet, III:cyan) composing this major association fiber system connect the frontal lobe with postrolandic parietotemporal association areas.

\section{Superior fronto-occipital fascicle (SFO):} the existence and location of the SFO were subject of continuous debate in the historical literature however, this fiber bundle is readily identifiable in DTI tractography. It is seen in the coronal plane as a triangular

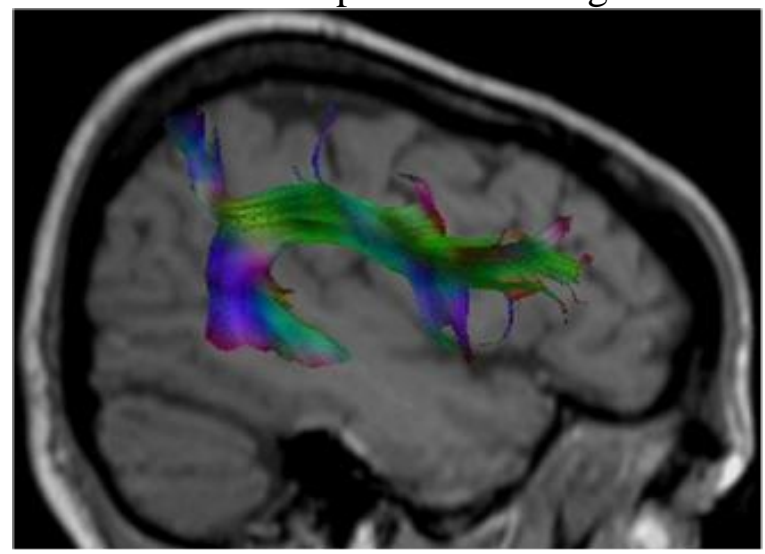

Figure 13: 3D, DTI reconstruction of the arcuate fascicle connecting the posterior ventrolatelar frontal lobe with the posterior part of the superior and middle temporal gyrus after arching around the lateral fissure.

fasciculus running above the caudate nucleus, medial to the coronal radiation and lateral to the fibers of the corpus callosum. It extends from the parieto-occipital junction to the dorsal prefrontal cortex connecting the superior parietal gyrus (parastriate areas 
important for peripheral vision and visual motion) with the dorsolateral prefrontal cortex of the middle and inferior frontal gyri (necessary for attention). These cortical connections make this tract relevant for visual spatial processing. (Fig 14)

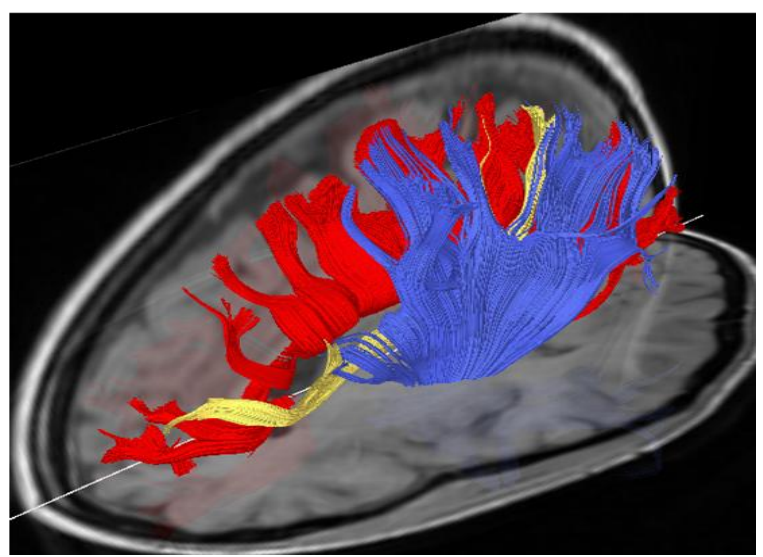

Figure 14: 3D, DTI reconstruction of the SOF fascicle (yellow). It is seem running medial to the coronal radiation (blue) and lateral to the fibers of the corpus callosum (red), extending from the parieto-occipital junction to the dorsal prefrontal cortex.

Cingulate fascicle $(\boldsymbol{C F})$ : lies within the white matter of the cingulated gyrus and extends from the frontal lobe, around the rostrum and genu of the $\mathrm{CC}$, above the body of the $\mathrm{CC}$, before curving ventrally around the spenium to lie within the white matter of the parahippocampal gyrus. It interconnects multiple areas around the CC including the orbital surface of the frontal lobe, the endorhinal and perirhinal cortex, the supplementary motor areas (SMA), the dorsolateral prefrontal cortex, the caudal inferior parietal lobule, the retrosplenial cortex, the parahippocampal cortex and the presubiculum (Fig 15). It forms the major component of the dorsal limbic pathway. By connecting the hippocampus and parahippocampal gyrus (critical for memory) with prefrontal areas (important for manipulating information, monitoring behaviour and for working memory) and the rostral cingulated gyrus (involved in motivation and drive), this fiber system is crucial for a wide range of motivational and emotional aspects of behaviour and for spatial working memory. Moreover, the limbic system plays a very important role in high-level mental processes relevant to memory and emotion. It is part of the Papez circuit that links the hippocampus, parahippocampal gyrus, mammilary bodies, thalamus, and cingulated gyrus. Other structures have subsequently been integrated into the limbic system including the amygdala, septal region and the olfactory bulb. The function of these structures has been implicated in dementia, epilepsy and schizophrenia.

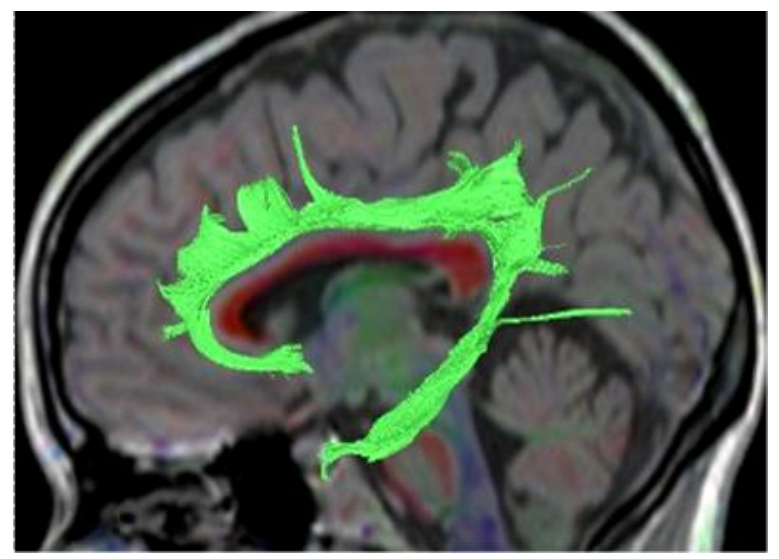

Figure 15: 3D, DTI reconstruction of the cingulated fascicle interconnecting multiple areas around the $\mathrm{CC}$ that are part of the dorsal limbic pathway.

Fornix: is also a limbic structure connecting the hippocampus with the hypothalamus. It is an association fiber tract however, it also has a small commissural component. Fibers arise from the hippocampus and parahippocampal gyrus of each side and run through the fimbria to join beneath the splenium of the $\mathrm{CC}$ to form the body of the fornix. Beneath the splenium, some fibrial fibers cross the midline to project to the contralateral parahippocampal gyrus and hippocampus forming the hippocampal commissure. Most of the fibers within the body run anteriorly, below the body of the CC, towards the anterior commissure. Above the interventricular foramen the body of the 
fornix divides into right and left columns. As each column approaches the AC it diverges again forming an anterior bundle, which curves posterior to the $\mathrm{AC}$ to enter the mammillary body of the hypothalamus as the postcommissural fornix, and an anterior bundle, which curves anterior to the $\mathrm{AC}$ and enters the hypothalamus as the precommissural anterior fornix (Fig. 16). Similar to the cingulum, the fornix is part of the dorsal limbic system and the Papez circuit having a very important role in highlevel mental processes relevant to memory and emotion.

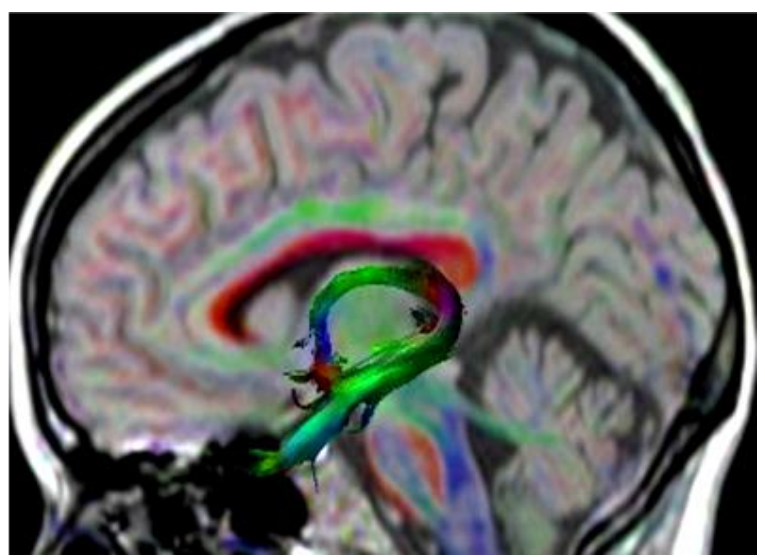

Figure 16: 3D, DTI reconstruction of the fornix connecting the hippocampus with the hypothalamus showing its course below.

Inferior longitudinal fascicle (ILF): first described by Burdach in 1822 and later by Dejerine represents a major occipitotemporal associative tract. Its presence has been challenged by evidence in non-human primates that connections between the two regions are entirely indirect, conveyed by the so called "occipito-temporal connection system"- a chain of U-shaped association fibers however, later evidence showed that the ILF is a major associative pathway distinct from fibers of the optic radiation and from U-shaped fibers connecting adjacent gyri. It arises in extrastriate visual association areas (cuneus medially,and fusiform and lingual gyri laterally), then it runs forward, parallel to the optic radiations and the tapetum, projecting to lateral (superior, middle and inferior temporal gyri) and medial (uncus, parahippocampal gyrus and amygdale) anterior temporal regions (Fig 17). No fibers are identified in the calcarine region. The ILF has a role in the ventral visual stream that is in object recognition, discrimination and memory. It appears to mediate the fast transfer of visual signals to anterior temporal regions and neuromodulatory back-projections from the amygdala to early visual areas. Face recognition is likely dependent upon the ILF. Disruption has been implicated in associative visual agnosia, prosopagnosia, visual amnesia, and visual hypoemotionality.

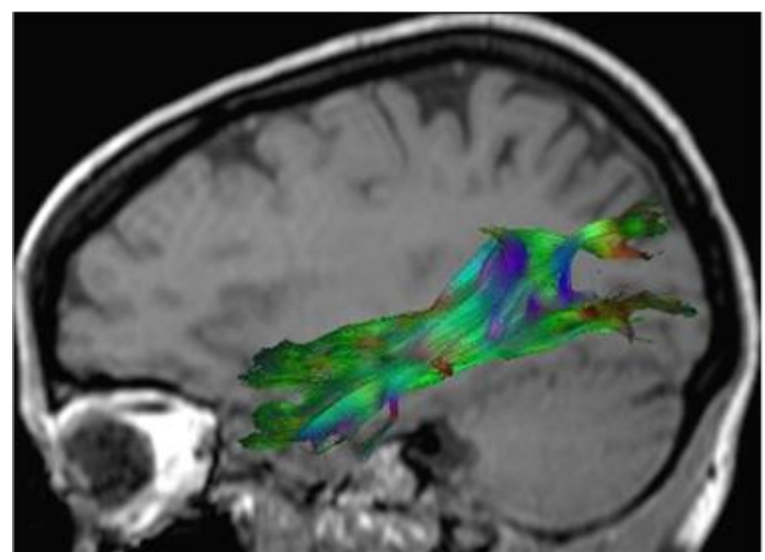

Figure 17: 3D, DTI reconstructions of the ILF, which is an important component of the ventral visual stream by connecting extrastriate visual association areas with medial and lateral temporal areas.

Inferior fronto-occipital fascicle (IFO): the existence of this fascicle in the primate brain has been debated in the recent literature although is often demonstrated in DTI tractography reconstructions. It runs posteriorly from mainly prefrontal cortical areas immediately superior to the uncinate fasciculus in the frontal lobe. At the junction of the frontal and temporal lobes, it narrows as it passes through the anterior floor of the external capsule and then continues posteriorly terminating in the middle and inferior gyri of the posterior temporal lobe and in the lingual and fusiform gyri of the 
inferior occipital lobe (Fig 18). This fascicle may also be a main component of the ventral subcortical pathway having an important role in object recognition, discrimination and semantic processing.

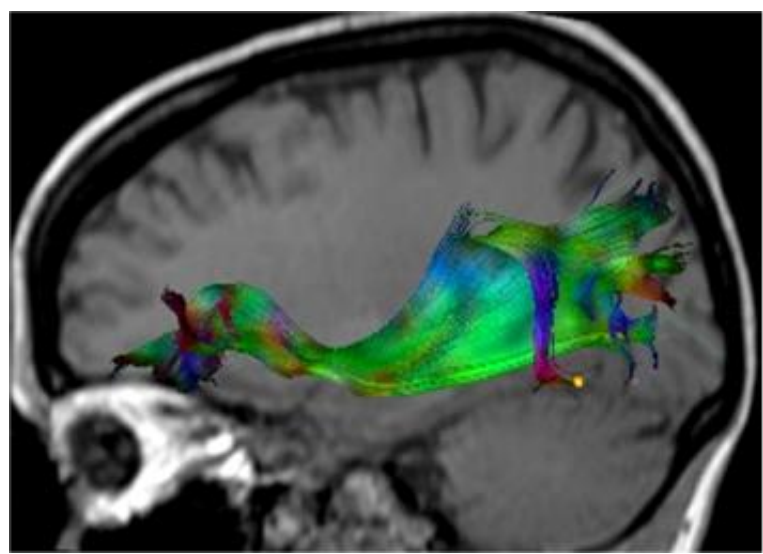

Figure 18: 3D, DTI reconstructions of the IFO, connecting prefrontal cortical areas with posterior temporal and inferior occipital cortical areas.

Uncinate fascicle: connects the anterior part of the temporal lobe with the orbital and polar frontal cortex. Dorsolateral fibers from the frontal pole run posteriorly to join with more ventral and medial fibers from the orbital cortex to form the uncinate fascicle, which runs for a short length inferior to the IFO before entering the temporal lobe as a single compact bundle. The uncinate then hooks anteromedially (through the limen insulae) to terminate in the temporal pole, uncus, hippocampal gyrus, and amygdale (Fig 19). The UF is a ventral limbic pathway. It links the rostral superior temporal gyrus (important for sound recognition), the rostral inferior temporal gyrus (important for object recognition) the medial temporal area (important for recognition memory) and the orbital, medial and prefrontal cortices(involved in emotion, inhibition and self regulation). It is critical for processing novel information, understanding emotional aspects of the nature of sounds and for self regulation, including the regulation of emotional responses to auditory stimuli. In other words the UF enables the interaction

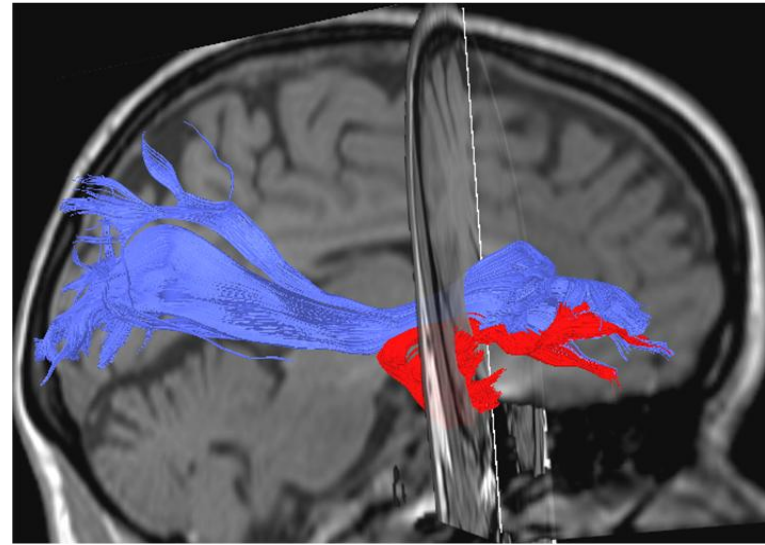

Figure 19: 3D, DTI reconstruction of the UF (red) showing its course between the orbital and polar frontal cortex and the anterior part of the temporal lobe and its anatomical relation with the IFO (blue). In its frontal portion the UF runs directly below the IOF and then separates from it as it hooks anteriomedially, at the level of the limen insulae, to enter the temporal lobe.

between emotion and cognition. It may also be critical in visual learning.

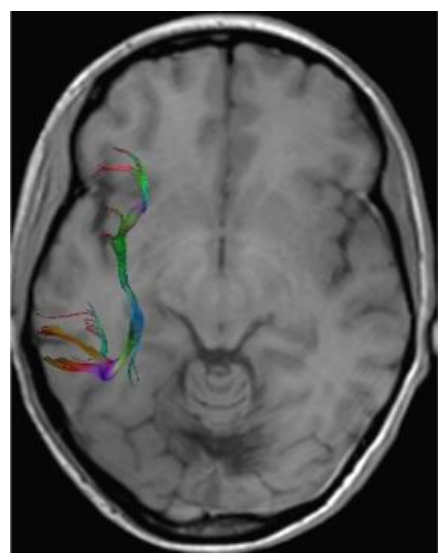

Figure 20: 3D. DTI reconstruction of the $\mathrm{EmC}$ situated between the claustrum and the insula, and interconnecting the mid-portion of the superior temporal region with ventral and lateral parts of the prefrontal cortex.

Extreme capsule (EmC): is situated between the claustrum and the insula, interconnecting the mid-portion of the superior temporal region with the mid portion of the ventral and lateral parts of the prefrontal cortex (Fig. 20). It is distinguished from the external capsule that is strictly a corticostriatal pathway. In the frontal lobe the EmC divides into a superior ramus that lies within the white mater of the inferior frontal lobe and 
an inferior ramus that lies beneath the claustrum on the floor of the orbital cortex, laterally adjacent to the UF. On the saggital images the EmC is seen to occupy a position behind the uncinate fasciculus in the superior temporal gyrus. By connecting the superior temporal cortex and insula with the orbital and dorsolateral prefrontal cortex may have a function in the connection between Broca's and Wernicke's areas important in the linguistic (non-articulatory) aspects of language communication. By virtue of its connections with the temporal operculum (auditory association cortices) may also be relevant to sound and language comprehension.

\section{Applications of DTI in the investigating connectivity of the human brain}

DTI has opened an entirely new window on the white matter anatomy with both clinical and scientific applications. It, has been applied clinically in neurosurgery for the presurgical mapping of WM tracts before intracranial mass resections, and already advanced the scientific understanding of many neurologic and psychiatric disorders. Its utility is found in both the localization and the quantitative assessment of specific neuronal pathways. The potential of this technique to address connectivity in the human brain has created a lot of excitement in the neuroscience communities. Studying diseased connectomes and their related dynamics is certainly going to significantly increase our understanding of the related pathophysiologies and potential therapeutic effects. However, there are certain methodological limitations that someone needs to be aware when considering the structural and dynamic complexity of mammalian brain. For example, despite recent progress, the biological basis of FA is not entirely clear. This measure is influenced by the degree of myelination, axon size, and axon density, integrity of axonal cell membranes in a complex way, but fiber path geometry and crossing fibers may also contribute. Several studies suggest that FA reflects valuable microstructural information that can be linked to electrophysiological measures of functional connectivity in a meaningful way however, this still needs further validation. The DTI method presently applied in humans has a limited resolution (both angular and voxel size) in comparison with classical invasive methods traditionally used to study connectivity. High field magnets and specialized acquisition techniques may seem promising in demonstrating intracortical terminations of axonal fibers (Fig. 21) however, still fall from being able to describe the complex cellular architecture of the human brain. Also none of the imaging methods so far provides directional information. The combination of DTI with MEG/EEG connectivity might provide directionality and dynamic information however, increasing effort must be made to merge the transdisciplinary competences required for such combinations. Therefore, clarification of the following questions is essential for transdisciplinary communication:

\section{Can we study connectivity of the brain using DTI?}

DTI provides information on the large scale structural substrate that supports coherent physiological activity and therefore, is probably the only non-invasive method available to provide in-vivo information on the anatomical networks (structural connectivity) connecting functional areas in the human brain. However, DTI does not provide information on large scale brain dynamics as it is recorded by electrophysiological and other functional neuroimaging techniques (functional connectivity) and also does not provide directional informational (effective 
connectivity). This distinction between the different types of connectivity is important in order to understand the limitations and contributions of the available brain mapping techniques in the investigation of the brain and its functions. Although the definition of a structural network helps us to understand the fundamental architecture of interregional connections, we must also consider functional networks and their directional links directly to understand information processing in the brain and mental representations.

\section{At which scale operates DTI to define structural connectivity?}

Brain networks are defined at different levels of scale that range from cells, to populations, to systems. They corresponde to levels of spatial resolution, which can be roughly categorized as microscale (micrometer resolution), mesoscale (corresponds to the spatial resolution of hundreds of micrometers), and macroscale (millimeter resolution). Connectivity at the microscale level comprises neural systems that are composed of interconnected neurons and mapping the human network composed by $10^{10}$ neurons and $10^{14}$ synaptic connections at this cellular resolution poses unique challenges that are beyond the resolution of DTI methods. Mesoscale connectivity is defined by anatomically and/or functionally distinct neuronal populations linking hundreds or thousands of individual neurons. Many anatomical structures in the brain contain similar local circuits (e.g., cortical columns). Methods for studying structural connectivity at the mesoscale level include cell staining and light microscopy, tract tracing using labelling agents, and electronomicroscopy reconstructions of sectioned tissue blocks. Studying structural connectivity at this level is also beyond the actual resolution of DTI methodology. At the macroscale level, large brain systems are parcellated into anatomically distinct areas that maintain specific patterns of interconnectivity or anatomically segregated brain regions connected by inter-regional pathways. Traditionally, connectivity at large scale has been mapped by destructive methods such as, histological dissection and staining, degeneration methods and by axonal tracing. A wide spectrum of diffusion imaging paradigms and computational tractography algorithms has been explored in recent years, which established DTI as promising new avenue for the non-invasive in vivo mapping of structural connectivity at the macroscale level. Further improvements in the spatial resolution of DTI may allow this technique to be applied in the near future for mapping connectivity also at the mesoscale level however, maps acquired through the use of diffusion imaging should allow for cross-validation with anatomical data collected by more classical histologic techniques. (Fig. 21)

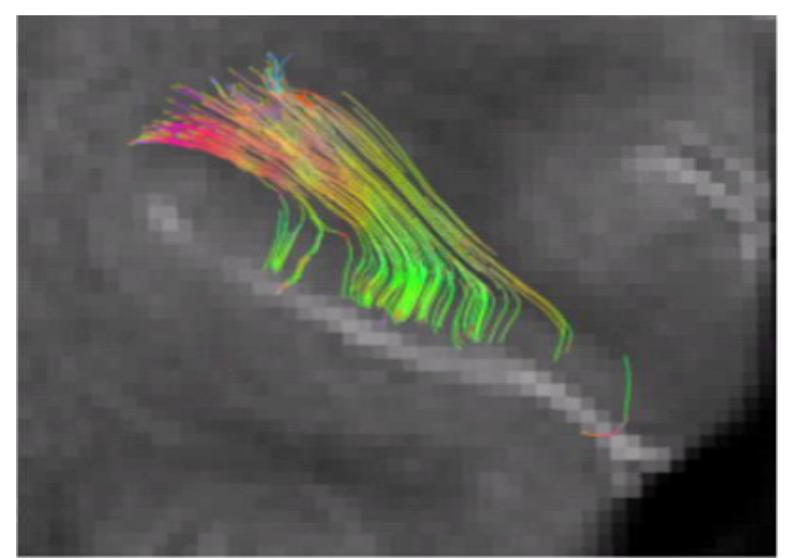

Figure 21: 3D, DTI high-resolution reconstruction of thalamocortical fibers acquired on a 3T MR system using specilized surface coils. The fibers are reconstructed up to the corticomedullary junction and eventually can be followed to the intracortical level.

\section{Suggested reading:}

1. M. Guye, et al. Curr Opin Neurol 2008; 21:393-403

2. N. Makris, et al. Cerebral Cortex 2005; 15:854-869 
3. Y. Assaf, et al. J Mol Neurosci 2008; 34:51-61

4. N. Makris, et al. NeuroImage 2007; 37:1100-1111

5. M. Catani, et al. Ann Neurol 2005; 57:8-16

6. L. Bonzano, et al. J. Neurscience 2008; 28:3227-3233

7. S. Hofer, et al. NeuroImage 2006; 32:989-994

8. D.S. Reich, et al. AJNR 2006; 27: 2168-2178

9. U. Bürgel, et al. NeuroImage 1999; 10:489-499

10. M. Catani. Curr Opin Neurol 2006; 19:599-606

11. M. Wahl, et al. J. Neurosci. 2007; 27:12132-12138
12. J.D. Schmahmann, et al. Brain 2007; 130:630-653.

13. V.J. Wedeen, et al. NeuroImage 2008; 41:1267-1277

14. P. Hagmann, et al. PLoS ONE 2007; 2(7):e597

15. E. Bullmore, et al. Nature Review Neuroscience 2009; 10:186-198

16. P. Stämpfli et al. JMRI 2006; 26:886893

17. P. Stämpfli, et al. Neuroimage 2006; 30:110-120

18. T. Jaermann, et al. Magn Res Med 2004; 51:230-236

19. P. Staempfli, et al NeuroImage 2008; 39:119-126

20. T. Järmann, et al. AJNR 2008; 29:146 - 150 